\title{
DIVISIBILITY OF ORDERED GROUPS
}

\author{
by I. W. WRIGHT
}

(Received 21st July 1971)

In this paper it is shown that divisibility of a complete lattice ordered (abelian) group is closely related to the existence of a sufficient number of small elements in the positive cone.

We shall denote the set of all real numbers by $R$ which symbol will be reserved for this purpose. All terms used are as defined in Birkhoff (1). For the reader's convenience we now define the two terms most used in the sequel.

Definition 1. Let $(G, \leqq)$ be a lattice ordered group. An element $c \in G$ is $a$ weak unit if both $c>0$ and $c \wedge|x|=0$ implies $x=0$.

Definition 2. A partially ordered group $(G, \leqq)$ is called integrally closed if $n a \leqq b$ for $n=1,2, \ldots$ implies $a \leqq 0$.

It is known (Fuchs (2), p. 90) that every complete lattice ordered group is integrally closed.

Lemma 1. Let $(G, \leqq)$ be a lattice ordered group. Assume that there exists a collection $S$ of weak units which has infimum zero.

Then if $y>0$ there exists $z>0$ such that $0<2 z \leqq y$.

Proof. Let $0<2 z \leqq y$. Since $(G, \leqq)$ is isolated it follows that $z>0$ and so

$$
0<z<2 z \leqq y \text {. }
$$

Thus if $0<2 z \leqq y$ holds for no $z$ then

$$
\lambda \in G: 0<\lambda \leqq y \Rightarrow 2 \lambda \text { 杰. }
$$

It follows that

for all such $\lambda$; for if

$$
(y-\lambda) \wedge \lambda=0
$$

then

$$
0<\mu=(y-\lambda) \wedge \lambda
$$

$$
0<2 \mu \leqq y .
$$

If $\lambda=y \wedge \phi$ with $\phi \in S$ then $0<\lambda \leqq y$, for $\lambda=0$ would imply $y=0$. So we can take $\lambda=y \wedge \phi$ in (1). This gives for all $\phi \in S,(y-y \wedge \phi) \wedge y \wedge \phi=0$, and since $\phi$ is a weak unit,

$$
(y-y \wedge \phi) \wedge y=0 .
$$

The left-hand side is $(y-\phi)^{+} \wedge y$; since $(y-\phi)^{+} \leqq y$ we get $(y-\phi)^{+}=0$, so

$$
0<y \leqq \phi
$$


for all $\phi \in S$, whence

a contradiction.

$$
0<y \leqq \bigwedge_{\phi \in S} \phi=0
$$

Lemma 2. Let $(G, \leqq)$ be a complete lattice ordered abelian group. Suppose $\left\{x_{i}: x_{i} \in G, i \in I\right\}$ is bounded above by an element $u$. Then for any positive integer $n$,

$$
n \bigvee_{i \in I} x_{i}=\bigvee_{i \in I} n x_{i}
$$

Proof. In the complete lattice ordered group, $x_{i} \leqq u$ and $n x_{i} \leqq n u$ for all $i \in I$, so that $\bigvee_{i \in I} x_{i}$ and $\bigvee_{i \in I} n x_{i}$ exist.

Now by the remark on p. 614 of Bernau (3),

$$
n \bigvee_{i \in I} x_{i}=\bigvee_{i \in I} n x_{i}
$$

Proposition 1. Let $(G, \leqq)$ be a complete lattice ordered group which has a collection $S$ of weak units whose infimum is zero. Then $(G, \leqq)$ is divisible by integers.

Proof. Let $n$ be a fixed positive integer and let $x$ be any point in $C^{*}$ (the strictly positive set of $(G, \leqq))$. We show that there exists $t \in C^{*}$ such that $n t=x$.

Form the set $T=\left\{w \in C^{*}: n w \leqq x\right\}$.

If $k$ is a positive integer such that $2^{k} \geqq n$ we see by $k$ applications of Lemma 1 that $T$ is non-empty. Now let $M$ be a maximal $\leqq$-chain of elements in $T$. Define $t=\sup M$.

We now show that $x=n t$. First we observe that $n t \leqq x$ (Lemma 2). Suppose $n t \neq x$. Then $k\left(2^{k} \geqq n\right)$ applications of Lemma 1 show that there exists $v \in C^{*}$ such that

$$
0<v<n v \leqq 2^{k} v \leqq x-n t
$$

It is easy to see that $n(t+v) \leqq x$ and that $(t+v)>0$. Thus $t+v \in T$. Now $t+v>\sup M$ and so $M \cup\{t+v\}$ is a chain in $T$ which contradicts our assumption that $M$ is a maximal chain. Thus $n t=x$.

It is clear that $t$ is unique since the group $G$ can contain no elements of finite order. We will denote $t$ by $\frac{x}{n}$.

Now let $g \in G$ be an arbitrary point. Since $(G, \leqq)$ is directed, $g=x_{1}-x_{2}$ where $x_{1}, x_{2} \in C^{*}$. Then we may (uniquely) define

$$
\frac{g}{n}=\frac{x_{1}}{n}-\frac{x_{2}}{n}
$$

Proposition 2. Let $(G$, $\leqq)$ be a complete lattice ordered group which is divisible by integers. Then $(G, \leqq)$ is a real vector lattice. 
Proof. Let $\lambda$ be any real number. Suppose $\left(\lambda_{i}\right)$ is a sequence of rationals which is convergent to $\lambda \in R$ in the usual topology. Now let $x$ be an arbitrary element of $G$. We can show that the sequence $\left(\lambda_{i} x\right)$ is order convergent (Birkhoff (1), p. 244) to a unique element of $G$. We shall denote this limit $\lambda x$.

Using the fact that $(G, \leqq)$ is integrally closed it is easy but tedious to show that $G$ is a unitary module over $R$ and that

$$
\begin{gathered}
\lambda \in R: \lambda>0, x \in G: x>0 \text { imply } \\
\lambda x>0 .
\end{gathered}
$$

Thus $(G, \leqq)$ is a real vector lattice.

Theorem. Let $(G, \leqq)$ be a complete lattice ordered group with weak unit. Then $(G, \leqq)$ is a real vector lattice if and only if $G$ contains a set of weak units whose infimum is zero.

Proof. Propositions 1 and 2 establish the "if" result. For the proof of the "only if " part let $u$ be the weak unit of $(G, \leqq)$, and form the set

$$
S=\left\{\frac{u}{n}: n=1,2,3, \ldots\right\} .
$$

The elements of $S$ are all weak units. Write $x=\inf S$. Clearly $x \geqq 0$. Since $n x \leqq u$ for every $n$, and $(G, \leqq)$ is integrally closed, $x \leqq 0$. Thus inf $S=0$.

\section{Acknowledgement}

The author wishes to thank Professor J. B. Miller for his helpful criticisms and suggestions. This work was done while the author held a Monash Graduate Scholarship.

\section{REFERENCES}

(1) G. BiRKhoff, Lattice Theory (3rd edition) (Amer. Math. Soc., 1967).

(2) L. Fuchs, Partially Ordered Algebraic Systems (Pergamon, 1963).

(3) S. J. BernaU, Unique representations of Archimedean lattice groups and normal Archimedean lattice rings, Proc. London Math. Soc. 15 (1965), 599-631.

MONASH UNIVERSITY

Clayton, Victoria 3168

AUSTRALIA

Now at:

Papua and New Guinea Institute of Technology

Box 793

LAE

New GuineA 